Thorax (1973), 28, 498.

\title{
Restoration of the strictured gullet ${ }^{1}$
}

\author{
R OW A N NICKS \\ Royal Prince Alfred Hospital, Sydney, Australia
}

\begin{abstract}
Nicks, R. (1973). Thorax, 28, 498-510. Restoration of the strictured gullet. Local plastic procedures which restore the lumen of the oesophagus are a satisfactory mode of treatment for patients with localized strictures, provided the remainder of the oesophagus is healthy and that a valvular sphincteric mechanism is restored.

Replacement of the irretrievably damaged oesophagus by an isoperistaltic graft of jejunum or colon in its original bed at a single operation is safe and satisfactory. In some circumstances retrosternal replacement is preferred. Subcutaneous placement is reserved for situations where viability is doubtful.

Experimental and clinical experience indicates that healthy isolated interposed grafts of the right length function well provided there is a free outflow from the stomach.

Grafts which are initially too short, or which have undergone terminal necrosis and are exteriorized to the anterior chest wall, will elongate in time, permitting subsequent retrosternal replacement and cervical anastomosis.

The terminal segment of oesophagus damaged by caustic stricture should be excised as a prophylaxis against cancer in later life.
\end{abstract}

Some problems encountered in treating strictures of the oesophagus are reviewed in the light of an experience which, although comparatively small, has been varied. The theme which now dominates this work is ways and means for preserving and restoring the natural gullet damaged by disease, provided this is not beyond repair.

This paper deals with conservative methods found to be helpful in dealing with long and short strictures at the cardia, and with techniques for replacement of an irretrievably damaged oesophagus. In addition, the problems of graft necrosis and subsequent elongation in the small and large bowel, a phenomenon which makes re-anastomosis possible at a later date, and that of carcinoma developing in caustic scars are each examined in the light of clinical experience.

\section{MATERIAL}

The data are drawn from clinical experience, supplemented by postmortem vascular contrast injection and radiographic studies and by dog experiments designed to investigate the behaviour of bowel loops connected with the stomach.

Four aspects, based largely on clinical experience, will be discussed, namely, (1) patients with hiatus hernia and fibrotic strictures; (2) a

1Presented to the Thoracic Society of Great Britain Summer Meeting at Cardiff 7-8 July 1972 miscellaneous group with long strictures and gros damage treated by replacement ; (3) graft elonga tion after necrosis; (4) carcinoma developing ing a caustic stricture.

HIATUS HERNIA AND REFLUX Twenty-six patientsi have been treated by a local plastic reconstruction of the stenosed lumen and provision of sphincteric mechanism at its junction with the stomach. Prior to surgery all patients had been. treated by antacids and dietetic measures designec to heal the mucosa.

Short strictures at the cardia The operation illustrated in Fig. 1 is suitable for localized fibrotie. lesions up to $1 \mathrm{~cm}$ in length in which the mucosh is mobile and pliable.

Following exposure and mobilization of the lower oesophagus by a left lower thoracotomy w bilateral vagotomy is performed above the cardias and the stricture is divided longitudinally by am incision extending into the healthy mucosa af either end, the site being indicated by a mercurse bougie. When the lesion is suitable and reconstruction can be done without undue tension, the mucosal lumen is restored using transversel $\vec{\Phi}$ placed interrupted Dacron sutures (Fig. 1) and the्ष suture line is reinforced by the gastric fundus o which is elevated and wrapped round the terminak gullet (Nissen and Rossetti, 1963). The associated 


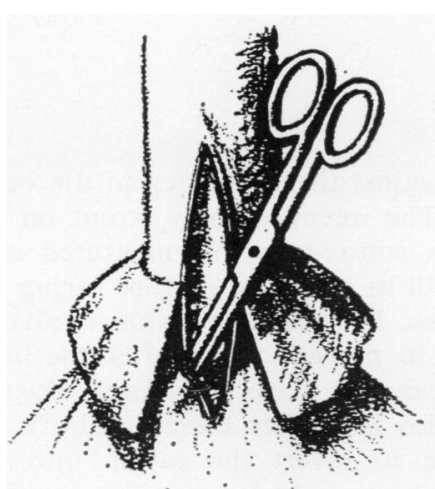

1.

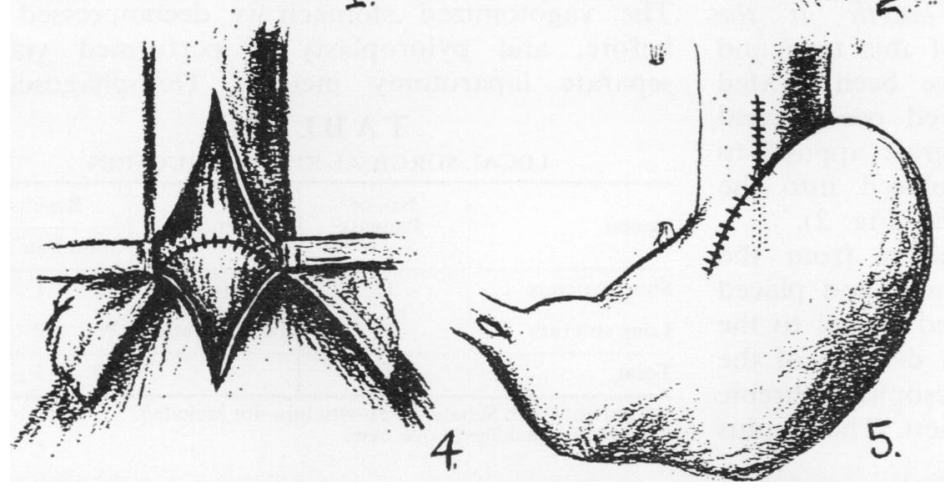

FIG. 1. Stages in the operation for short stricture.
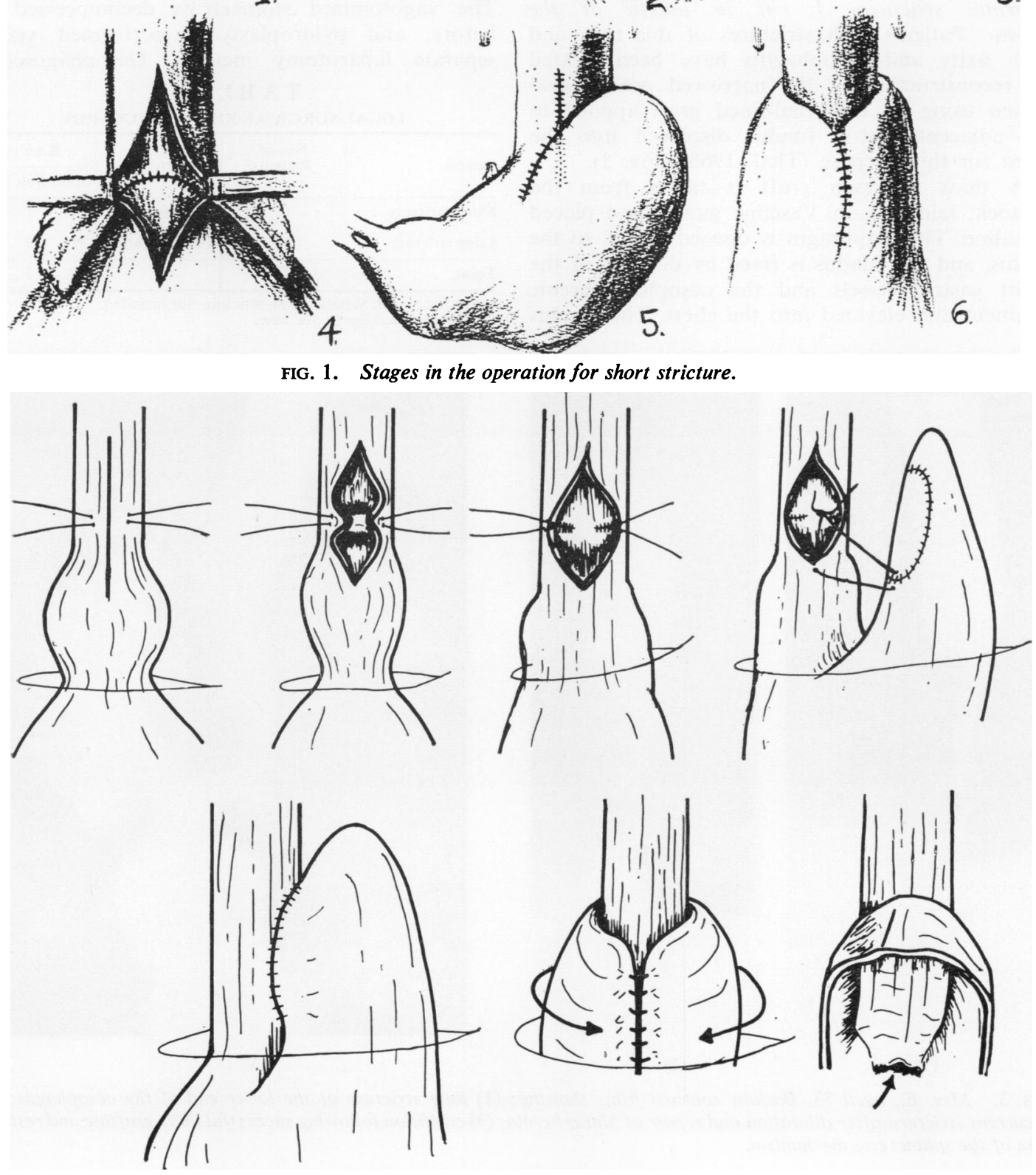

3.

6.

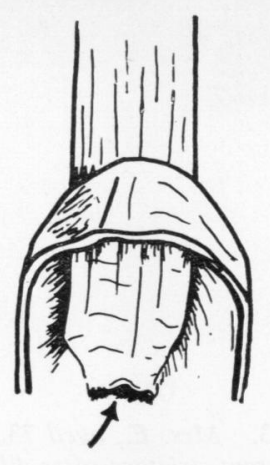

FIG. 2. Stages in the operation for long stricture (Thal). 
hernia is reduced by adaptations of the Allison technique, provided shortening is minor; but when this is marked and reflux has been controlled by fundoplication, abdominal replacement of the hernia is unnecessary.

Decompression of the stomach by means of a nasogastric tube sutured to the nasal ala as a precaution against its withdrawal is considered to be of great value in prophylaxis against leakage.

Fibrotic strictures $1 \mathrm{~cm}$ in length at the cardia Patients with strictures of this type and with fixity and oesophagitis have been treated by reconstruction of the narrowed oesophageal lumen using an epithelial-lined graft applied to the adjacent gastric fundus displaced into the chest for this purpose (Thal, 1968) (Fig. 2).

A thick Thiersch graft is taken from the buttock, laid out on Vaseline gauze, and placed in saline. The diaphragm is opened lateral to the hiatus, and the fundus is freed by division of the short gastric vessels and the oesophagophrenic ligament and elevated into the chest. The fundus

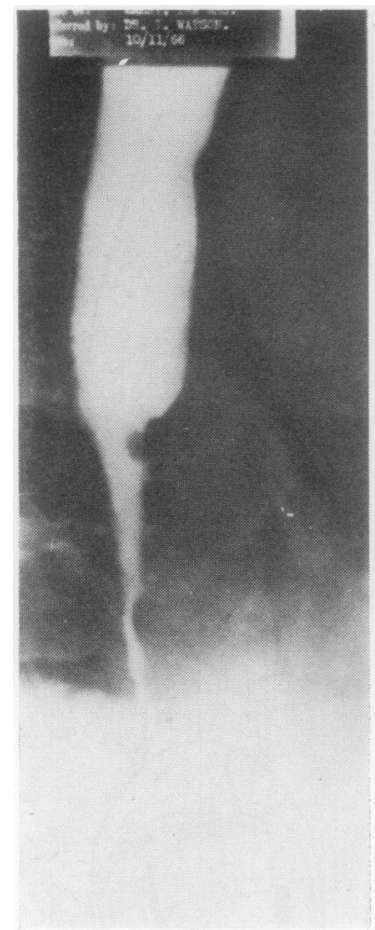

(1)

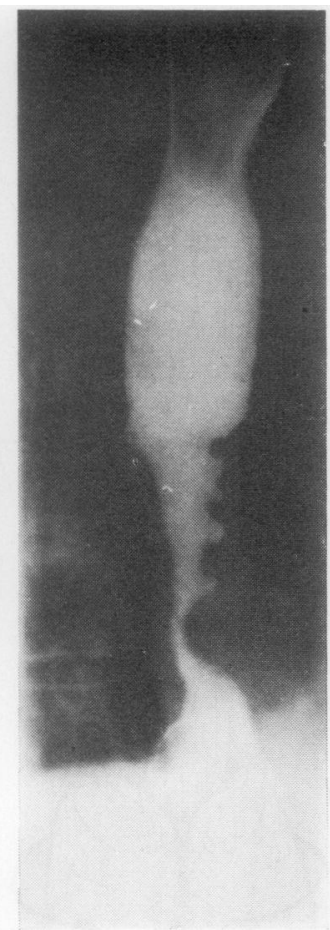

(2) is manipulated against the deficiency in the oeso phageal lumen. The intended inlay is cut on the large size and is sutured to this measured area음 of the fundus with its epithelial surface facing the oesophageal lumen. In order to provide a valvulan mechanism, and to prevent reflux after the linedy inlay has been sealed in place by running Dacron sutures, the fundus is further elevated above the repair and made to invert the cardia into the stomach and make a valvular papillary orifices The vagotomized stomach is decompressed as:्र before, and pyloroplasty is performed via a separate laparotomy incision. Oesophagoscopy

\begin{tabular}{l|c|c|cc}
\multicolumn{3}{c}{ T A B L E I } \\
LOCAL SURGICAL RECONSTRUCTION
\end{tabular}

${ }^{211}$ patients with Schatzki web-stricture not included.
${ }^{2}$ Temporary leakage in one case.

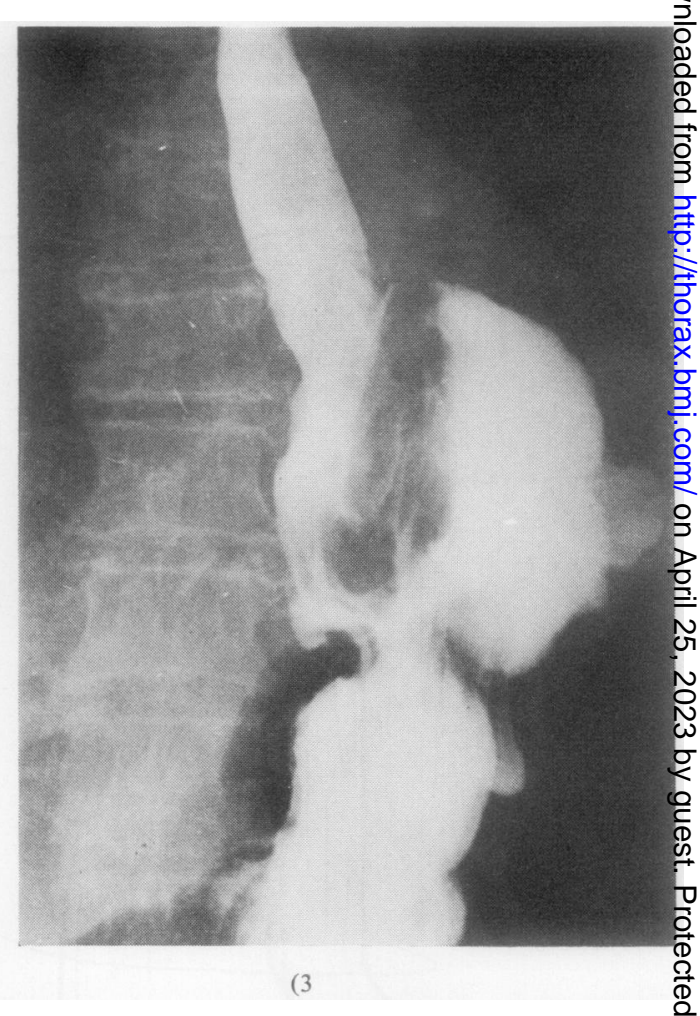

FIG. 3. Mrs. E., aged 73. Barium contrast films showing: (1) long stricture at the lower end of the oesophagus; (2ذे recurrent stricture after dilatation and repair of hiatus hernia; (3) condition following successful inlay grafting and restoras tion of the sphinct eric mechanism. 
and gentle bouginage is routinely done before discharge from hospital.

Great care is exercised to maintain the lumen at the time of reconstruction of the valve mechanism and to elevate the fundus well above the inlay repair.

The mucosal reconstruction of short strictures has been successful in restoring the lumen and a sphincteric mechanism in 14 out of a total of 15 carefully selected patients (Table I).

One patient, a 51-year-old doctor, died following later perforation of a mucosal ulcer at the site of the pylorotomy incision. The ulcer was one of many superficial gastric ulcers found to be present at necropsy. At a follow-up of the survivors it was ascertained that all swallowed without difficulty and usually without reflux. Oesophagoscopic inspection revealed healthy mucosa at the site of repair. Oesophagofundoplastic (Thal, 1968) reconstruction with inlay-grafting for long and intractable strictures was successfully performed on 11 patients, three of whom were in their seventies (Fig. 3). Normal swallowing with only minor reflux was achieved, but two patients presented a minor recurrence of symptoms. A temporary leakage occurred in one patient, an elderly psychopath who withdrew his nasogastric tube on the night of repair despite the firm suture fixing it to his nose, but the end result following temporary intestinal feeding for two weeks was good (Fig. 4).

Before the procedure was elaborated, younger patients with long strictures were treated by resection and jejunal or colonic interposition, and older patients by periodic dilatations or resection and oesophagogastrostomy. We have not performed replacement for this type of lesion for two years in the older group although prior to this time it was standard treatment. The longterm results will need careful assessment.

MISCELLANEOUS GROUP REQUIRING RESECTION AND REPLACEMENT Replacement has been reserved for patients with an irretrievably damaged gullet in whom local reconstruction was not considered applicable. The methods employed were oesophagogastrostomy, jejunal interposition and colonic replacement.

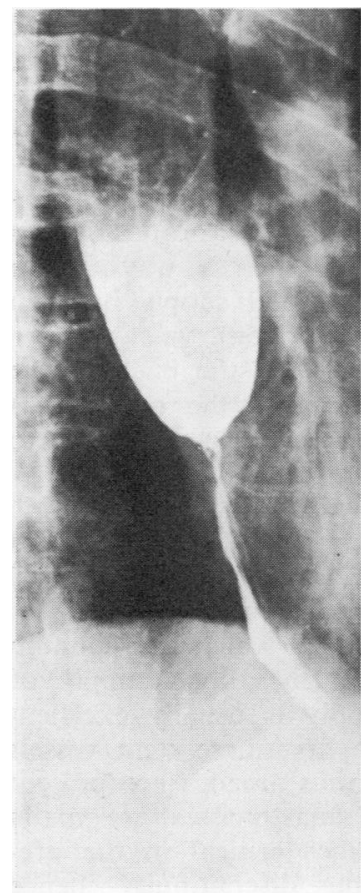

(1)

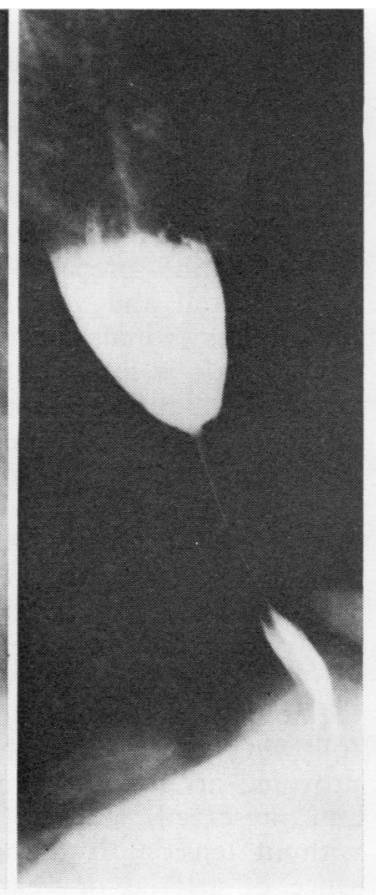

(1)

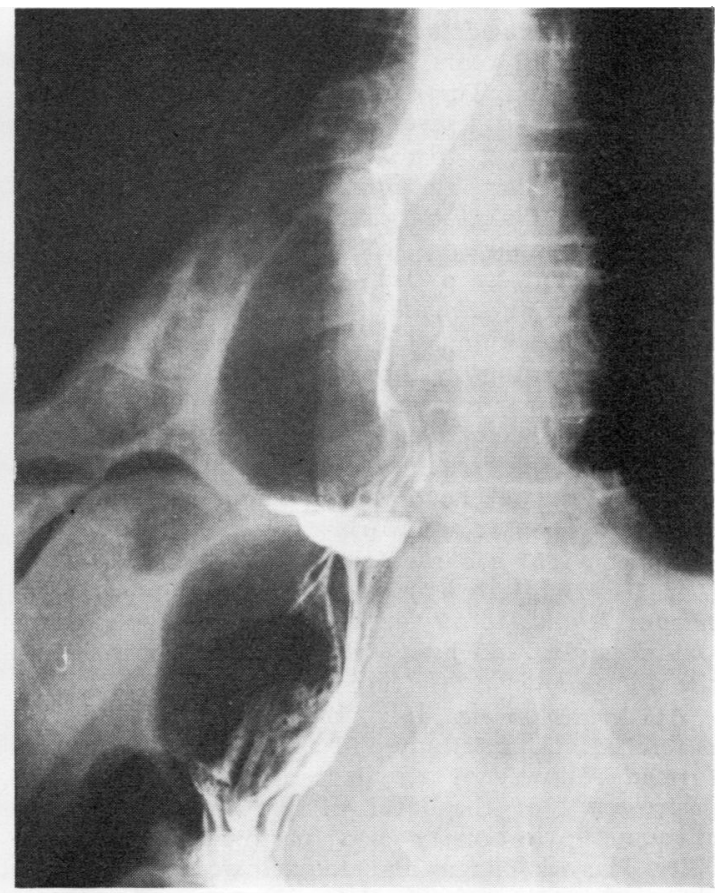

(2)

FIG. 4. Male aged 68 years, a psychotic: (1) before operation revealing a rigid stricture; (2) after Thal inlay-grafting showing restoration and healing after a temporary leakage. 
Oesophagogastrostomy The high reconstruction from the right side (Lewis, 1947) is now reserved for elderly patients with strictures of recent onset in the vicinity of the aortic arch, and is not employed in younger people nor in patients with caustic strictures.

The operation was performed in patients early in the series before late complications were fully appreciated and other alternatives available. Although there are many exceptions oesophagogastrostomy has usually been followed by a recurrence after a variable period of time in patients with long-standing symptoms of oesophagitis and stricture. In three children on whom I performed this operation 20 years ago for strictures associated with hiatus hernia from infancy, and in one adult with a caustic stricture extending to the thoracic inlet, anastomotic strictures recurred which were eventually successfully treated by colonic interposition. However, in particular cases this is a valuable operation.

Case 1 was a 61-year-old retired inspector who had complained of periodic epigastric pain after meals and at night for 18 years. This was relieved by vomiting and by antacids. He had experienced remissions for periods of a year at a time. A duodenal ulcer had been demonstrated by repeated barium studies. For 10 years he had suffered recurrent episodes of purpura. Three years before admission to hospital he had had a severe haematemesis and melaena while undergoing treatment with steroids for the purpura. Further bleeding episodes were repeated in 1967 and 1968. At the time of admission in 1968, he had continual epigastric pains, accompanied now by reflux and by a deep boring retrosternal pain. These pains were made worse on eating but were relieved by vomiting. Radiographic examination following a barium swallow at this time showed a normal oesophagus, a small hiatus hernia with reflux, a normal stomach with no delay in the passage of barium, and an irritable, irregular duodenal cap. A few months later he began to vomit once or twice daily and he gave up work.

In June 1969 a barium swallow showed a stricture, 4 in $(100 \mathrm{~mm})$ in length, in the mid-thoracic oesophagus, leading to a fixed hiatus hernia. The stomach was distended and little barium passed onwards into the duodenum.

At operation in July 1969, the stomach was mobilized at laparotomy and a pyloroplasty was performed, followed by a right transthoracic oesophagogastrostomy at the level of the aortic arch. An adequate pyloroplasty was performed with difficulty. The oesophagus and stomach were separated by sharp dissection from the vascular and fibrotic mediastinal tissues.

The strictured area was excised, the cardia oversewn, and a high oesophagogastrostomy performed. The fundus was wrapped round the anastomosis and buried within the mediastinum. Aspiration by mear of a nasogastric tube (maintained in position by 0 stitch through the ala of the nose) kept the stomact empty of contents until its tone returned. Despite severe postoperative exacerbation of the purpur⿸丆口 early severe renal failure, and pulmonary problems the patient made a good recovery. Six months late he had regained his weight, looked well, enjoyed his food, and had no dysphagia nor reflux. The barium study revealed a free passage of barium through the stomach to the duodenum. No ulcer was demore strated. He remains well three years later.

Comment: Oesophagitis and subsequent stri⿻ ture formation developed in this patient over $\stackrel{\infty}{\mathbb{2}}$ period of six months following pyloric obstruction and bouts of vomiting. The reactivation of the ulcer which led to the patient's subsequer? troubles was provoked by cortisone.

At the time of admission he was gravely with oesophageal and pyloric obstruction and would not have withstood a replacement. The operation which was done relieved the obstruction at both sites. The woody-hard mediastinitis which enveloped the oesophagus and extended upwark along its length to the upper mediastinum wag noteworthy.

Jejunal replacement This operation is preferres for replacements extending to the aortic arch The colon is used as an equally safe and satis factory alternative method at this level and above

The oesophagus and upper jejunum are a proached through a left thoraco-abdominal inct sion and the loop selected for interposition examined and prepared in such a way that good arterial and venous blood supply betwee the arcades is maintained (Fig. 5a).

The graft is inserted between the back of the stomach, about its middle, and the oesophagus above the stricture, which is displaced lateral to the aortic arch for this purpose (Fig. 5b).

Several problems relevant to jejunal grafting having been studied, namely, (1) arterial an venous blood supply; (2) peristalsis and blinse loops ; (3) graft necrosis and elongation:

The limitation of height to which the jejunump can be taken depends on the blood supply of the loop. The blood supply is usually excellent following division of the first three stem vesse provided arterial and venous arcades are preserot and preserved, and the anastomoses are made without tension. It becomes critical in the pre sence of arterial or venous discontinuity-hence the necessity of examining these vessels on bot sides of the mesentery before ligature. The venous drainage is more critical in longer grafts exten ing into the neck, where it may be necessary to 


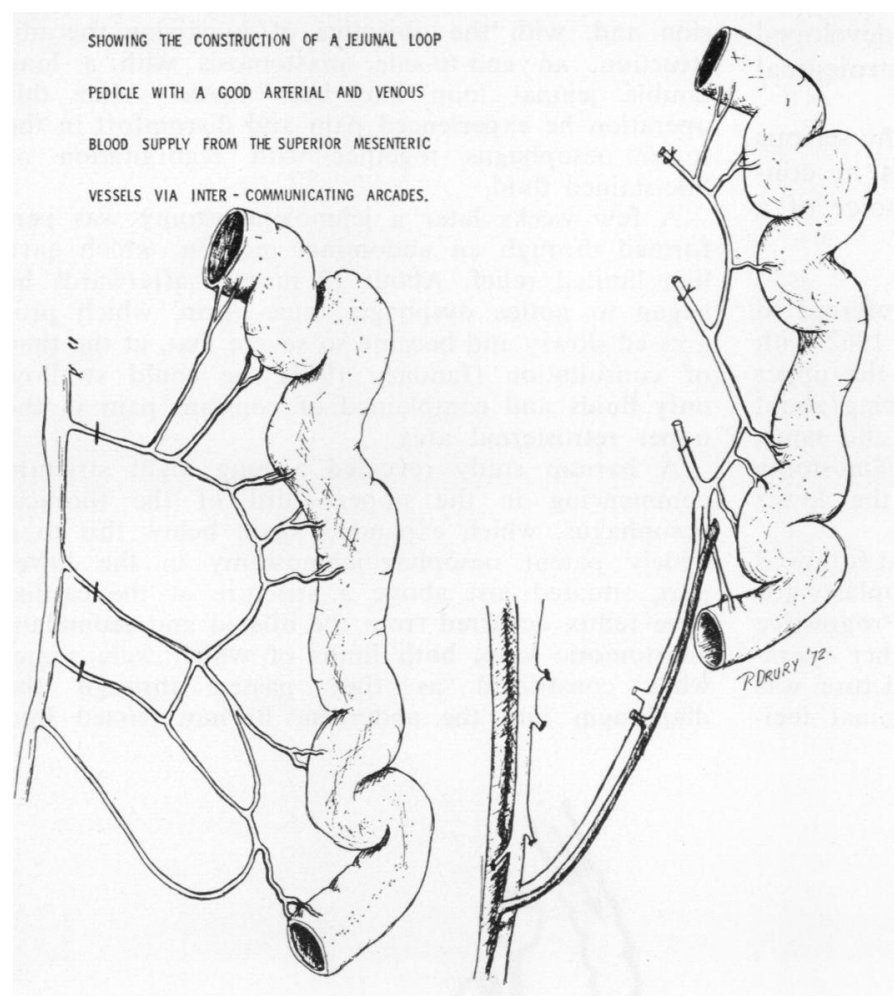

(a)

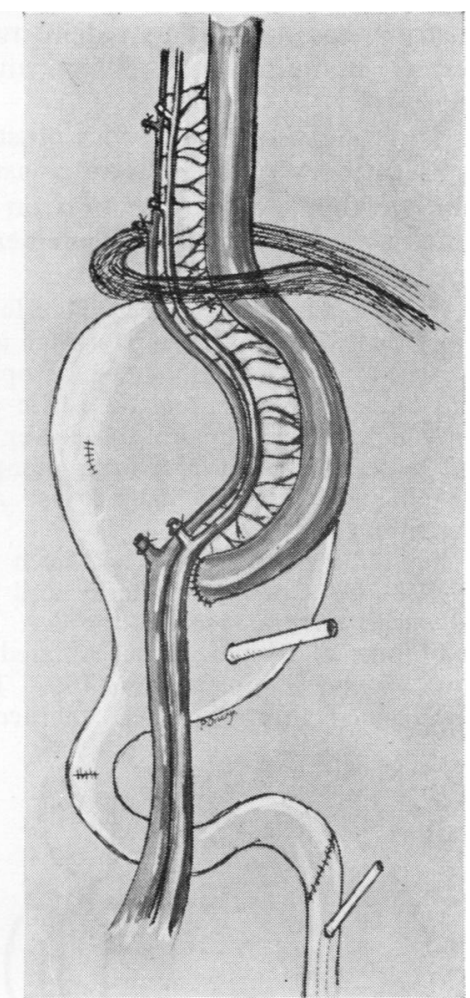

(b)

FIG. 5. (a) Construction of a jejunal loop. Three stem vessels have been ligated and divided. In the right-hand drawing both the artery and vein to the pedicle are pictured without tension or torsion of the mesentery. (b) Interposition operation. Jejunal graft in place with pedicle. There is no redundancy in the chest. After pylorotomy the stomach is decompressed and a temporary feeding jejunostomy has been inserted.

divide four or five stem vessels to provide sufficient length (Ong, 1971). The possibility of congestive necrosis occurring may be indicated by placing temporary clips on the lower vessels before making ligations, and by taking the greatest care to avoid injury or torsion of the thin-walled vessels.

Whereas an isoperistaltic interposition graft between oesophagus and stomach, serving as a conduit, functions well for an indefinite period, blind loops, and long loops remaining in continuity with the jejunum, do not function and become the site of ulceration and stricture at the site of anastomosis.

In a series of dog experiments the mucosa of isolated loops connected with the stomach, whether exteriorized to skin or made into a blind loop or into a double gastric loop, showed no evidence of inflammation.

In contrast, all the animals in which the jejunal loop connected with the stomach remained in continuity with the small bowel by a Roux-en-y anastomosis at its distal end, and so subject to continual reflux of bile and activated digestive juices, developed ulceration and stenosis of the jejunum adjacent to the anastomosis.

Clinical susceptibility to ulceration following this method of reconstruction after gastric resection was reported by Lambley (1962).

In several patients in whom the loop was not completely isolated, or in whom antiperistalsis occurred in a blind loop, there was gross interference with nutrition and recurrent stricture formation. In one patient a long redundant antiperistaltic loop was the cause, in another a long jejunal loop had been anastomosed to the greater curvature of the stomach at the time of colonic replacement of the strictured oesophagus. At the time he had active duodenal ulceration with pyloric stenosis complicated by a scarred non-functioning 
gastroenterostomy. This patient rapidly developed severe malnutrition, reflux, and gastrojejunal stenosis.

The following case history illustrates the serious consequences of long loop anastomosis in continuity, and focuses attention on the choice of a particular approach to replacement.

Case 2 was a man aged 51 . He was referred to the Royal Prince Alfred Hospital in July 1962 with two fibrotic strictures of the oesophagus, the upper situated at the level of the aortic arch being about one inch in length and the lower much the same, at the cardia. A double-loop oesophagojejunostomy opening was widely patent just above the lower stricture (Fig. 6).

In 1940 a hiatus hernia had been repaired followed in 1954 by a pneumonectomy and thoracoplasty for advanced tuberculosis of the left lung. Progressive symptoms of dysphagia necessitated a further operation on the oesophagus in 1957. The stricture was approached through a left thoraco-abdominal inci- sion and, with the objective of bypassing the ob-倞 struction, an end-to-side anastomosis with a longo double jejunal loop had been made. After this 0 operation he experienced pain and discomfort in the lower oesophagus together with regurgitation of $\overrightarrow{\mathbb{D}}^{\vec{\alpha}}$ bile-stained fluid.

A few weeks later a jejunojejunostomy was per-ê formed through an abdominal incision, which gave $\overrightarrow{-}$ him limited relief. About 12 months afterwards hebegan to notice dysphagia once again, which pro- $\vec{\omega}$ gressed slowly and became so severe that, at the timeo of consultation (January 1963), he could swallow only fluids and complained of constant pain in the
upper retrosternal area.

A barium study revealed a long, tight stricture? commencing in the upper third of the thoracicit oesophagus, which expanded again below this to $\mathrm{a}^{\infty}$ widely patent oesophagojejunostomy in the lower part, situated just above a stricture at the cardia.Free reflux occurred from the dilated and redundant anastomotic loop, both limbs of which were some what constricted as they passed through the $\overrightarrow{0}$ diaphragm into the abdomen. Barium passed into

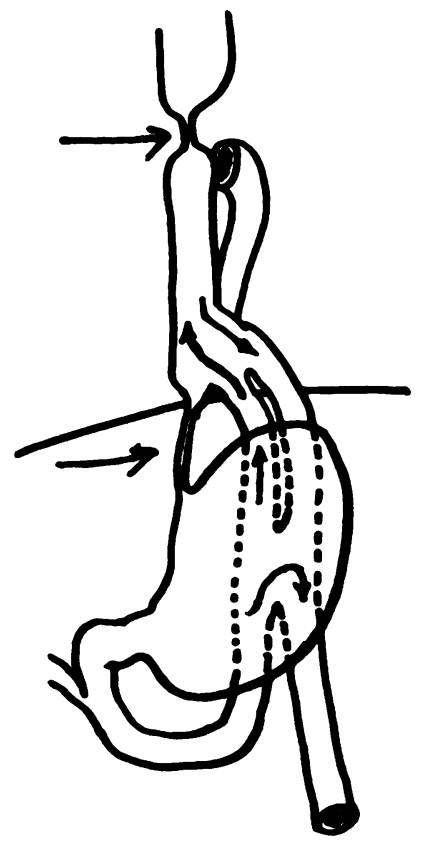

High Reflux Stricture following previous Oescophag - Jajunostomy for low Stricture

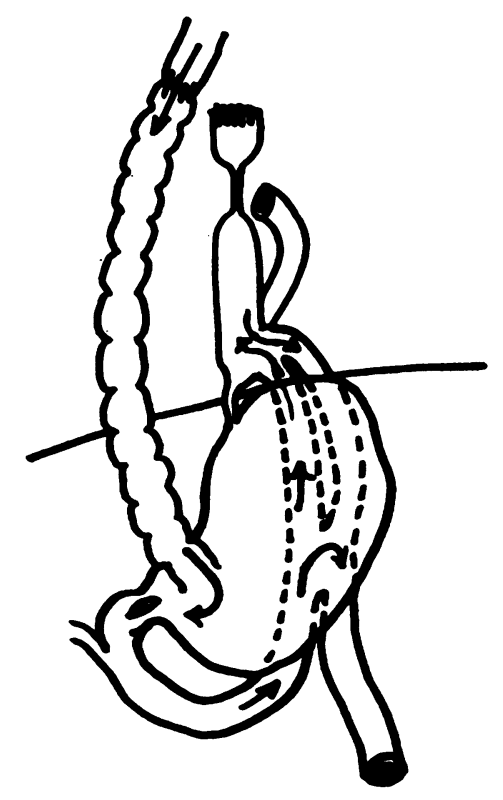

Restoration

by Retrosternal Colonic Interposition

(b)

FIG. 6. Case 2. (a) Line drawing of barium studies showing bypass of a stricture with a double jejunal loop. Despite jejunojejunostomy, between the limbs, reflux led to a high stricture at the aortic arch (top arrow). (b) Following colonic interposition. 
the dilated jejunum through the efferent limb, but none entered the afferent limb. At oesophagoscopy the oesophageal lumen was filled with an alkaline bile-stained fluid. The thickened mucosa was healthy above a tight, acutely ulcerated stricture which bled profusely when dilated.

Although he had obstructive airways disease in his single remaining lung, his vital capacity of 2.13 litres $(54 \%)$ was thought to be adequate to withstand operation provided the right pleural cavity was not entered.

A retrosternal bypass of the entire thoracic oesophagus was carried out using the right colon. The caecum was anastomosed to the upper end of the divided oesophagus, the lower end being oversewn and left in the mediastinum.

The patient made a rapid recovery from this operation and when examined in January 1972, nine years later, he was without symptoms.

Comment: A retrosternal colonic replacement, leaving the lower oesophagus intact, was the only option. The institution of unobstructed swallowing, gastric digestion, and the passage of the chyme through the duodenum and intestine completely restored nutrition to normal, substantiating the general experience that this sequential flow is necessary for healthy digestion.

Colonic replacement Isoperistaltic interposition of the colon as a replacement of long strictures is a satisfactory operation at all levels within the thorax and neck.

This single-stage operation is performed through a left lower thoraco-abdominal incision which permits of choice between the methods and replacement in the normal posterior mediastinal bed. The measured colonic segment, pedicled on the left colic artery and vein, provides an adequate length for the highest replacements.

In certain circumstances, namely when the strictured area is high or when the left-sided approach has been complicated by a previous major operative procedure (case 2), anterior retrosternal interposition is preferable. Petrov's (1959) general technique may be followed as a single-stage procedure. The right colon together with the caecum and a few inches of the terminal ileum are mobilized and the ileocolic vessels divided through a long laparotomy incision. The cervical oesophagus is isolated in the left side of the neck. After dividing the sternomastoid muscle and excising the inner end of the clavicle, a new bed is fashioned by passing the right hand behind the sternum to the neck.

The caecum and terminal ileum are carefully withdrawn into the neck with the caecum elevated on its right side and a single layer anastomosis is performed between the terminal ileum and the divided oesophagus, its distal end being closed and allowed to retract well down into the posterior mediastinum.

After performing a pyloroplasty, the colon is divided at a suitable length and the graft is anastomosed with the anterior wall of the stomach ; intestinal continuity is restored by an ileotransverse colostomy. Two serious problems associated with colonic interposition are infection, and congestive necrosis of the graft due to venous obstruction. Infection will not be discussed except to indicate that we have followed Belsey (1965) in relying on mechanical emptying of the colon at the time of surgery and that we routinely perform the anastomosis in the neck for lesions sited at the aortic arch level and above.

Necrosis is usually due to venous obstruction for the marginal artery provides a good anastomotic arterial supply as far down as the descending colon, apart from an occasional deficiency in the communicating arcade between the middle colic and left colic arteries at the splenic flexure (Fig. 7). If the left colic vessels are to be used as a pedicle, the adequacy of the marginal artery should be checked by temporarily clamping the middle colic pedicle while the splenic flexure is dissected free. There is constant good venous drainage of the transverse and descending colon into the inferior mesenteric vein via the left colic artery. On the right side, venous intercommunications via a marginal vein are lacking between the ileocolic vein, a separate right colic vein, and the middle colic in 15 to $20 \%$ of patients. As a corollary, the patency of marginal veins should be retested before division.

If the terminal ileum, which has a good lumen and a free arterial and venous blood supply, is used for the cervical anastomosis with the oesophagus, and the caecum and ascending colon are brought up retrosternally for this purpose, there is valvular unobstructed swallowing (Petrov, 1959).

Apart from minor infections, which will not be discussed, the results of colonic interposition (Table II) have been satisfactory in 11 of the 12 patients. The single unsatisfactory result occurred in a patient who had a gastrectomy at the same time for active duodenal ulceration and restenosis of a previous gastroenterostomy. It was due not to the colonic graft, which functioned admirably, but to malnutrition, reflux, and restenosis of the stomach outlet following a gastrectomy and reconstruction by a long antiperistaltic loop which passed to the greater curvature of the stomach. 


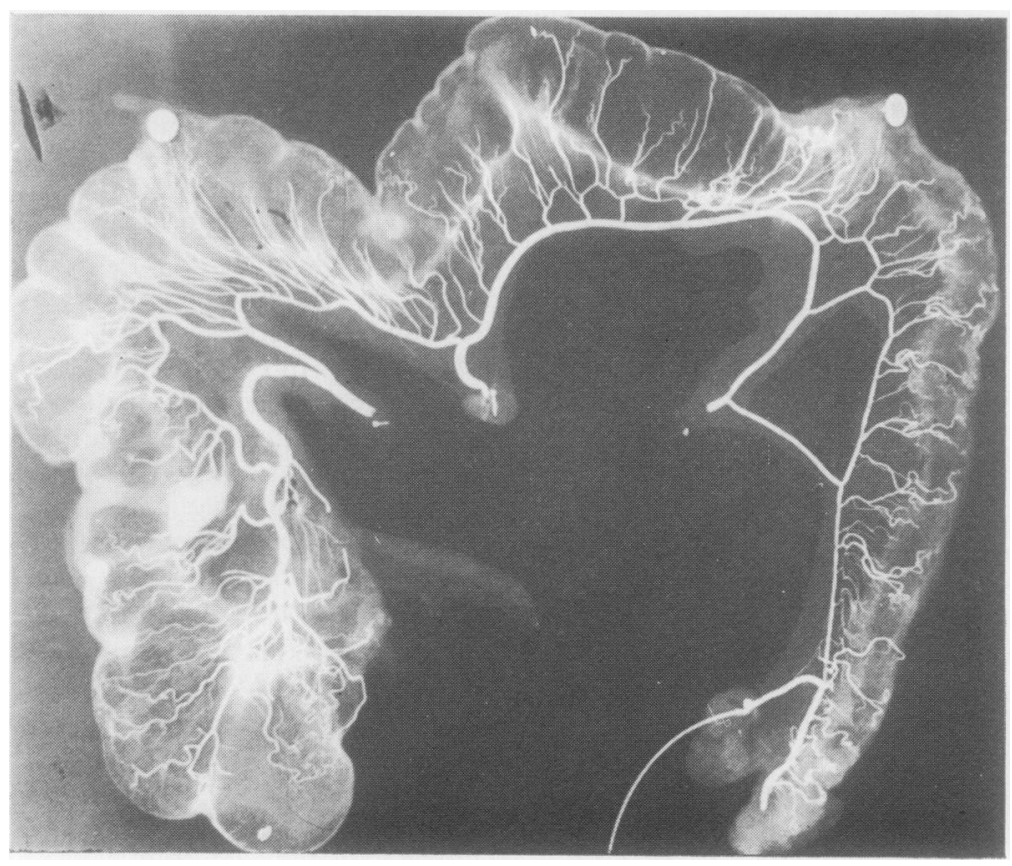

FIG. 7. Barium gelatine contrast study of colonic arteries. The ileocolic, middle colic, and inferior colic arteries intercommunicate freely by marginal branches.

T A B LE I I

BENIGN OESOPHAGEAL STRICTURE 1950-72 RESECTION AND RECONSTRUCTION

\begin{tabular}{|c|c|c|c|c|c|}
\hline \multirow{2}{*}{ Treatment } & \multirow{2}{*}{ No. } & \multicolumn{4}{|c|}{ Results } \\
\hline & & Good & $\begin{array}{l}\text { Necrosis } \\
\text { Fistula }\end{array}$ & Recurrence & Death \\
\hline $\begin{array}{l}\text { Oesophago- } \\
\text { gastrostomy } \\
\text { Jejunal }\end{array}$ & 10 & 6 & 0 & 4 & 0 \\
\hline $\begin{array}{l}\text { replacement } \\
\text { Colonic }\end{array}$ & 11 & 8 & 1 & 1 & $1^{*}$ \\
\hline $\begin{array}{l}\text { replacement } \\
\text { Lye } \\
\text { Miscellan- } \\
\text { eous }\end{array}$ & ${ }_{7}^{5} 12$ & 11 & 1 & 0 & 0 \\
\hline $\begin{array}{l}\text { Late end- to- } \\
\text { end anasto- } \\
\text { mosis } \\
\text { following } \\
\text { necrosis }\end{array}$ & 2 & 2 & 0 & 0 & 0 \\
\hline Total & 35 & 27 & 2 & 5 & 1 \\
\hline
\end{tabular}

GRAFT NECROSIS AND SUBSEQUENT ELONGATION AND HYPERTROPHY OF THE INTRA-ABDOMINAL LOOP In the following two patients there was failure of the graft following postoperative necrosis. Despite this, successful retrosternal interposition with an anastomosis at the root of the neck was subsequently possible.
Case 3 A woman aged 27 was admitted to the Royal Prince Alfred Hospital in 1963 complaining of life-long dysphagia which had been treated by repeated dilatations. A barium contrast study revealed a long stricture of the lower third of the oesophagus extending to the aortic arch.

In 1962 she had been treated for Graves' disease with a course of carbimazole and later by a subtotal. thyroidectomy. She was considered euthyroid at theo time of her current admission although exophthalmos was present.

The stricture was exposed via a left thoracoabdominal incision and a jejunal interposition waso performed at the aortic arch level. A week following this procedure she became hyperpyrexic and ill. Her pulse rate was $100 /$ minute. The graft was explored and found to be doubtfully viable at its upper end? The cervical oesophagus and the graft were exw teriorized. She made a quick recovery but became thin and wasted from malnutrition. An early attempto at closure failed because of tension between the endsunderlying the thin excoriated skin, which had beens damaged by reflux from the stomach, and because of her general state of malnutrition. The excoriation later became so unbearable that the two ends were्ष separated for 4 inches $(100 \mathrm{~mm})$ by a skin flap, and oral feeding was carried out by joining both end $\$$ with a large plastic tube (Fig. 8a).

She was highly nervous, apprehensive, and thyror 


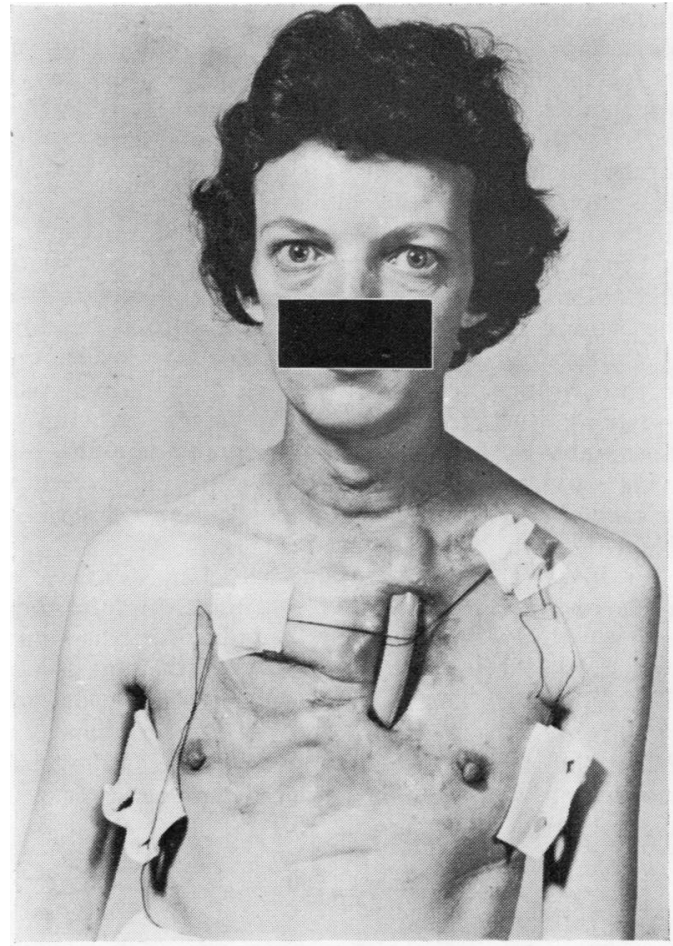

(a)

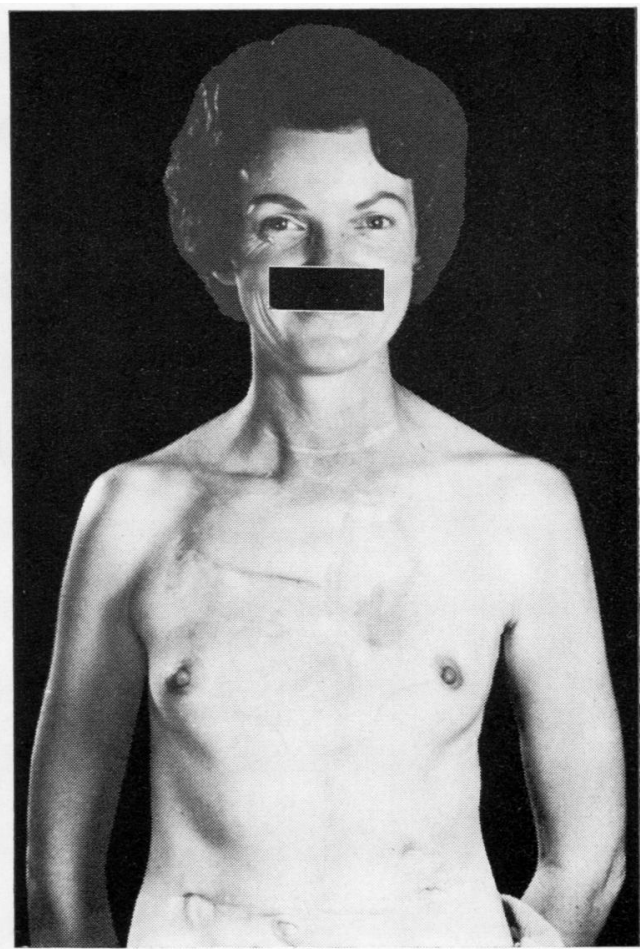

(b)

FIG. 8. Case 3 (a) Following necrosis and shortening of the jejunal graft swallowing was restored by a prosthetic tube. Note wasting and thyrotoxicosis. (b) Following direct anastomosis at the root of the neck seven years later.

toxic, and reluctant to have further surgery. Seven years elapsed before her confidence was restored to the extent that the inconvenience and discomfort of her condition outweighed the fear of an operation.

At laparotomy in August 1970 it was found that the jejunal loop in the abdomen and its mesentery had elongated considerably. It was not adherent and the anastomosis opened without inflammation into the stomach. It was possible to free the loop, and with the added length provided by elongation to withdraw this into the neck for anastomosis with the gullet. This was done without tension following excision of the inner end of the clavicle, the surrounding tissues giving the anastomosis good support. The wound healed satisfactorily. At present she swallows normally and her nutrition is so improved that she is $42 \mathrm{lb}(19 \mathrm{~kg})$ heavier than before operation (Fig. 8b).

Case 4 A man aged 43 was admitted to the Royal Prince Alfred Hospital in 1963 with extensive strictures of the lower oesophagus and pylorus from swallowing hydrochloric acid some months previously. A gastroenterostomy was performed for the pyloric stricture, and the oesophagus was managed by dilata- tions. These were not successful in managing the oesophageal stricture and replacement was decided upon.

In 1965 a retrosternal interposition of the right colon was performed between the cervical oesophagus and the anterior wall of the stomach (Fig. 9(2)).

This operation was followed by immediate serious complications of mediastinitis, a lung abscess, and an abdominal abscess, all of which were consequences of necrosis of the caecum. These areas were drained successfully, but a total oesophageal obstruction resulted, and this necessitated permanent gastrostomy feeding.

He expectorated saliva into a bowl which he carried everywhere. He had been fed by the gastrostomy for six years and at the time when he was referred to the Thoracic Clinical Unit (RN) for consideration of a further attempt at reconstruction.

On examination gross scarring was present at the root of the neck and in the abdomen. It was considered that reconstruction should be attempted, but little hope was held that the large bowel could be used for this purpose. Besides being very short following extensive sloughing of the caecum it was the seat of chronic ulcerative colitis. 


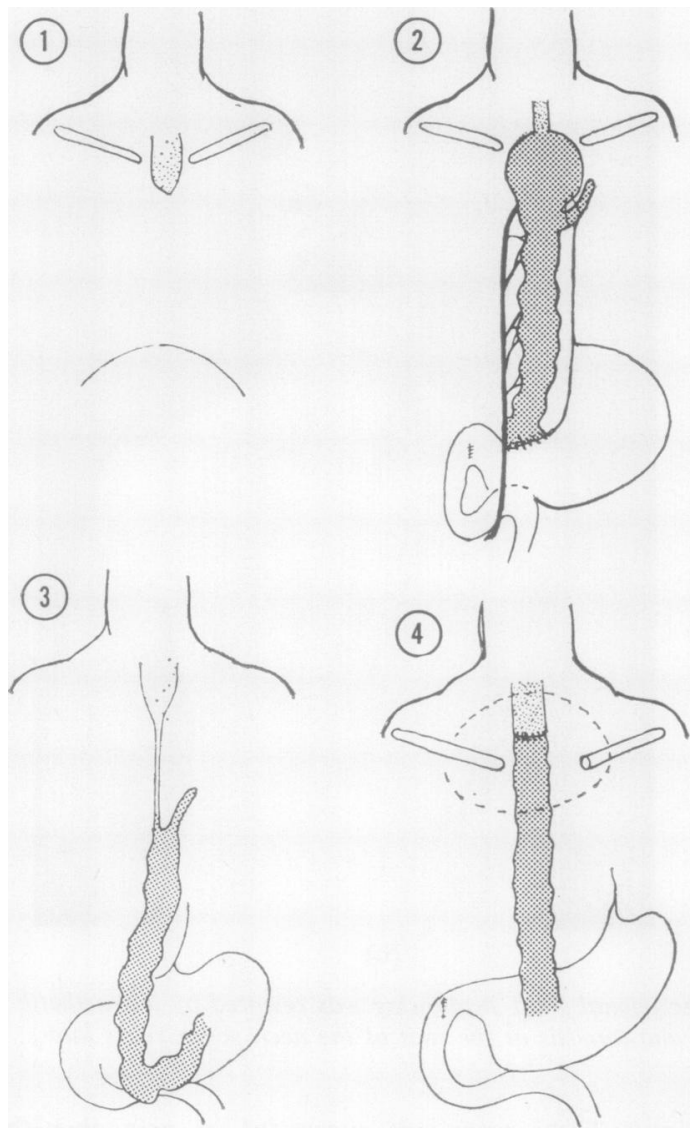

FIG. 9. Case 4. Drawing shows (1) complete oesophageal stenosis following ingestion of acid; (2) after retrosternal colonic replacement; (3) postoperative necrosis of caecum and ascending colon with elongation of abdominal segment; (4) direct end-to-end suture in root of neck.

The neck was explored through the anterior cervical scar and the oesophagus was isolated. It was traced to a ropy scar which continued behind the sternum (Fig. 9(3) ). When the abdomen was opened it was found impossible to free the jejunum because of dense adhesions resulting from previous operations. The doubtful large bowel loop, which was now regarded as a possible replacement graft, opened widely and apparently without any inflammation into the anterior wall of the stomach. It appeared to have elongated since the previous operation and, when followed upwards into the thorax by an anterior sternotomy, it was found to terminate in woody scar tissue about 3 inches $(76 \mathrm{~mm})$ below the manubrium. The appendix was still intact.

The thoracic colon was patently dissected from the densely fibrotic mediastinum. When free, it could be withdrawn into the neck by straightening the elongated and hypertrophied abdominal loop and 0 its vessels. The appendix was excised and, as the 음 upper end of the colon was friable, the adjacent $\overline{\bar{\alpha}}$ ends of the colon and the oesophagus were brought $\overparen{\nabla}$ to the surface and sutured side by side to the skin. $\stackrel{2}{2}$ The sternum and the abdomen were closed as a $ळ$ single incision drained its upper and lower ends. $\vec{\circ}$

Ten days later the open ends of the oesophagus and colon were anastomosed together. Postoperatively $\vec{\omega}$

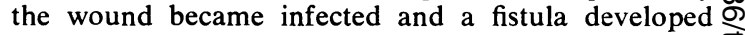
at the site of the anastomosis due, it was thought, $\overrightarrow{\vec{x}}$ to the chronic ulcerative colitis and to the scar tissue overlying the anastomosis. A further attempt at $\infty$ closure by excising the ends and replacing the over- $\rightarrow$ lying scarred cervical tissues with a previously fashioned vascular delayed pedicle flap of skin was $\infty$ decided upon.

In July 1971, the oesophagocolic anastomosis was made using $3 / 0$ Deknatel sutures with the knots $\subseteq$ tied outwards, and the skin pedicle flap was rotated $₹$ and sutured into position overlying the anastomosis $\overrightarrow{0}$ (Fig. 9 (4) ). The wound healed. $\mathrm{He}$ was soon swallowing normally for the first time in eight years. When the gastrostomy had been closed he was discharged home. He has remained well ever since and returned to work (Fig. 10).

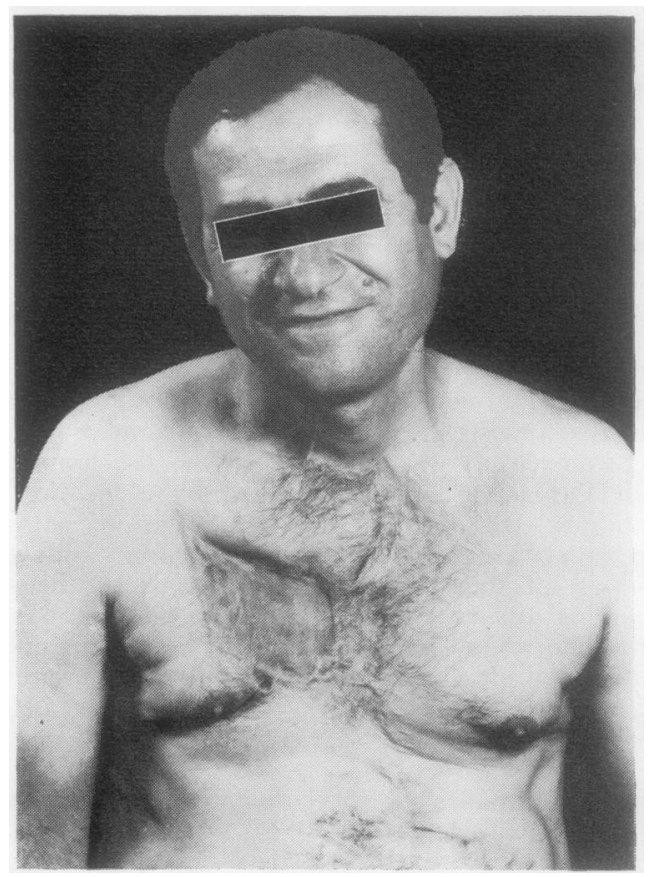

FIG. 10. Case 4. Final result after excision and anastomosis. 
Comment: 1. It is probable that ulcerative colitis played a minor part in the necrosis of the caecum and ascending colon. It is interesting to note that the chronic colitis was still present and the bowel inflamed seven years after isolation from all continuity with the colon. This has not interfered with its new function.

2. Seven years after extensive sloughing, withdrawal of the colonic graft into the neck was made possible by natural elongation and hypertrophy of the free abdominal colon and its mesentery.

3. Failure of the initial re-anastomosis was due to both dense scarring of the skin and surrounding tissues and to chronic ulcerative colitis. Success followed when the tissues were covered by a large healthy vascular delayed-pedicle skin flap.

CARCINOMA DEVELOPING IN A CAUSTIC STRICTURE An association with scar cancer, similar to that occurring in skin damaged by deep burns, was noted by Benedict (1946) and is now generally accepted.

The development of such a carcinoma in a patient with a life-long caustic stricture following colonic bypass is recorded as indicating the need for excision of the strictured area.

Case 5 A woman aged 43 was admitted to the Royal Prince Alfred Hospital with intractable dysphagia associated with an extensive stricture of the thoracic oesophagus in April 1960. The condition followed accidental swallowing of caustic soda when she was 1 year old. A gastrostomy was performed at the time, through which she was fed for 25 years. The tube was then removed, for she was able to swallow liquids and soft foods by mouth. She married and had a family. The oesophageal stricture, which was dilated periodically over the next 18 years, had become impassable at the time of admission.

A barium swallow revealed an almost total oesophageal obstruction beginning at the upper border of the aortic arch.

In April 1960 a retrosternal isoperistaltic right colonic bypass was performed at a single session, the lower anastomosis being made with the anterior wall of the stomach, and the upper between the caecum and the divided oesophagus, the lower end of which was oversewn and dropped back into the superior mediastinum.

Following this operation she made a good recovery and was able to swallow normally for the first time in her life. She gained $28 \mathrm{lb}(12.7 \mathrm{~kg})$ in weight and was happy.

She returned 20 months later in June 1962 with recurrent dysphagia. Oesophagoscopy revealed a constriction at the upper anastomosis, biopsy of which revealed a well-differentiated squamous carcinoma. She died on 10 December 1962.

\section{DISCUSSION}

In our clinic localized benign strictures of the gullet have been successfully treated by one of several techniques, viz., dilatation, local plastic procedures, and various types of reconstruction.

Attitudes to problems and details of techniques have evolved with experience. The present emphasis is on conservation whenever possible, illustrating the wisdom of Barrett's (1962) dictum that 'the oesophagus is a specialized viscus which cannot be replaced by any graft that performs its function perfectly'.

The power of resolution of digestive oesophagitis and severe obstructive oedema when reflux is abolished, by restoration of a valvular mechanism at the cardia, has been observed by many authors (Allison, 1951; Collis, 1961 ; Hayward, 1961; Nissen and Rosetti, 1963 ; Harrison and Gompels, 1971).

It has been my experience that established strictures are intractable and recur after dilatation and simple reduction procedures. The reconstructive operations reported (vide supra) for this type of lesion accept that the strictured area must be resected together with its mucosal lining, and the suture line protected from digestion by a valvular mechanism. The scope of this concept has now been enlarged to include most strictures under the aortic arch, but this will need to be re-assessed in the future.

In individual patients the choice of an operation for replacement of an oesophagus irretrievably strictured over a long area by caustic or by other diseases is highly personal and based on experience.

Our operations are carried out at a single stage, and the graft is replaced in the original bed of the excised oesophagus, except in special circumstances.

No surgeon, except Belsey (1965), has an experience comparable with that of Yudin (1944), who, by exploiting the Roux (1907) jejunal loop, provided a key to the successful management of extensive lye strictures in patients from all over Russia at a time before chest surgery had been developed, and before blood and electrolyte replacement and antibiotic therapy had become routine in major surgery. Petrov (1959) has carried on and developed this work. He employs the retrosternally transplanted right colon and anastomoses the terminal ileum with the oesophagus. Yudin demonstrated that a long isoperistaltic loop of jejunum with a suitably prepared mesentery supporting the freely communicating arterial and venous arcades without tension could 
be safely transferred to the neck subcutaneously and form a bridge between the gullet and the stomach, bypassing strictures situated even at the pharyngeal level. He performed his operations under a local anaesthetic, carefully preparing a jejunal segment with a mesentery sufficiently long to avoid tension on its vessels and closed at its upper end. He pushed the jejunum up through a wide subcutaneous tunnel prepared by means of long-handled bougies made for the purpose.

The maintenance of a good blood supply to jejunal and colon grafts is of paramount importance to successful transplantation. Studies of jejunal blood vessels and clinical experience indicate that the flow through intercommunicating arcades of arteries and veins is always adequate after division of two or three stem vessels, but it becomes increasingly critical as more are divided. This is more apparent in the veins, patency of which should be verified on both sides of the mesentery by inspection and dissection before the lower stems are divided (Ong, 1971).

The intercommunicating arterial blood supply to the colon via the marginal artery is always adequate except for a doubtful arcade at the splenic flexure, but the marginal veins are sometimes deficient ( 15 to $20 \%$ ) between the ileocolic, a separate right colic, and the middle colic vein (Nicks, 1967).

When the blood supply is doubtful and when necrosis of an intrathoracic graft occurs, tragedy may be averted by employing Yudin's staged subcutaneous operation (case 3 ). If the jejunal or colonic segment is not of sufficient length, or when it has been shortened by necrosis, an anastomosis with the cervical oesophagus may be successfully accomplished after an interval of time. This is made possible by elongation and hypertrophy of the free abdominal loop (cases 3 and 4).

In order to avoid the hazard of necrosis, the jejunum is chosen for short replacements extending to the aortic arch level, and the colon when extra length is required. Isolated loops of jejunum or colon are not subject to digestive erosion provided a free outlet exists from the stomach. In the presence of obstruction, or reflux of digestive juices, our experience and experimental observations indicate that stenosis is likely to recur.

Antiperistaltic loops of jejunum and colon have been unsatisfactory in Australian experience, and isoperistalsis is a cardinal requirement for success.
Much of the clinical work has been referred from the A. W. Morrow Department of Gastro-Enterology and the Physicians of the Royal Prince Alfred Hospital.

The radiology was studied with the help of the Department of Radiology, Royal Prince Alfred Hospital and the pathology at the Fairfax Institute of Pathology.

The illustrations were done by the Department of Medical Illustration, Royal Prince Alfred Hospital.

This study was aided by a grant from the Royal Prince Alfred Hospital Research Fund.

\section{REFERENCES}

Allison, P. R. (1951). Reflux esophagitis, sliding hiatal hernia, and the anatomy of repair. Surgery, Gynaecology and Obstetrics, 92, 419.

Barrett, N. R. (1962). Benign stricture in the lower esophagus. Journal of Thoracic and Cardiovascular Surgery, 43, 703.

Belsey, R. (1965). Reconstruction of the esophagus with left colon. Journal of Thoracic and Cardiovascular Surgery, 49, 33.

Benedict, E. B. (1946). Benign stricture of the esophagus. Gastroenterology, 6, 328.

Collis, J. L. (1961). Gastroplasty. Thorax, 16, 197.

- (1968). Surgical control of reflux in hiatus hernia. American Journal of Surgery, 115, 465.

Harrison, G. K., and Gompels, B. M. (1971). Treatment of $\frac{\circ}{\square}$ reflux strictures of the oesophagus by the Nissen-Rossetti operation. Thorax, 26, 77.

Hayward, J. (1961). The treatment of fibrous stricture of the oesophagus associated with hiatal hernia. Thorax,16,45.

Lambley, D. G. (1962). The case against gastrectomy with $\mathrm{y}$-anastomosis in treatment of peptic ulcer. British Medical Journal, 1, 1588.

Lewis, I. (1947). The surgical treatment of carcinoma of the oesophagus. British Journal of Surgery, 34, 18.

Nicks, R. (1967). Colonic replacement of the oesphagus; some observations on infarction and wound leakage. British Journal of Surgery, 54, 124.

Nissen, R., and Rossetti, M. (1963). Surgery of the cardia ventriculi. Ciba Symposium, 11, 195.

Ong, G. B. (1971). Resection and reconstruction of the esophagus. Current Problems in Surgery, Sept. 1971, 3. 음 - (1972). Personal communication.

Petrov, B. A. (1959). Retrosternal artificial esophagus from $\mathrm{N}$ jejunum and colon. Surgery, 45, 890.

Roux, C. (1907). l'Oesophago-jejuno-gastrostomose; nouvelle opération pour rétrécissement infranchissable de l'oesophage. Semaine Médicale, 27, 37.

Thal, A. P. (1968). A unified approach to surgical problems of the esophagogastric junction. Annals of Surgery, $168,542$.

Yudin, S. S. (1944). The surgical construction of 80 cases + of artificial esophagus. Surgery, Gynaecology and Obstetrics, 78, 561 . 